\title{
Using technological frames as an analytic tool in value sensitive design
}

\author{
Christiane Grünloh ${ }^{1,2}$ (1)
}

Published online: 27 June 2018

(c) The Author(s) 2018

\begin{abstract}
This article proposes the use of technological frames (TF) as an analytical tool to support the investigations within value sensitive design. TF can help to identify values that are consistent or conflicting within and between stakeholders, which is exemplified with a case of patient accessible electronic health records in Sweden. The article concludes that TF can help to identify values, which may then help to understand and address possible concerns in the design process.
\end{abstract}

Keywords Value sensitive design · Technological frames $\cdot$ eHealth $\cdot$ Patient empowerment $\cdot$ Patient accessible electronic health records

\section{Introduction}

Technological developments and innovations affect not only an individual's personal life but also society in general. It has been acknowledged that our changed and much more complex relationship with computers provokes new questions, for which a broader approach is needed that explicitly addresses human values (Sellen et al. 2009).

Value sensitive design (VSD) is such an approach that aims to account for human values throughout the design process by integrating conceptual, empirical, and technical investigations (Friedman et al. 2006). VSD has been described as an interactional theory, meaning that while certain design features might support or hinder certain values, the actual use depends on the goals of people interacting with it (Friedman et al. 2006, p. 361).

Orlikowski and Gash (1994) developed the concept of technological frames (TF) as a systematic approach to examine people's interpretation of a particular technology. In their definition, TF concern assumptions, expectations, and knowledge that people use to understand technology.

Christiane Grünloh

grunloh@kth.se

1 School of Electrical Engineering and Computer Science, KTH Royal Institute of Technology, 10044 Stockholm, Sweden

2 Institute of Informatics, TH Köln University of Applied Sciences, 51643 Gummersbach, Germany
While not explicitly including values in their framework, their approach builds on and extends research into "the cognitions and values of users and designers" (Orlikowski and Gash 1994, p. 174), for example the influence of designers' perceptions and values on the systems development. Human values are often implicated in system design (cf. Friedman et al. 2006, p. 364f), thus these values influence and inform also the interpretation of the technology. In their empirical studies, Orlikowski and Gash (1994) identified three core domains of the participants' TF: (1) Nature of Technology — what the technology is, (2) Technology Strategy — why it was introduced, and (3) Technology in Use-how it is used to create various changes in work. This initial set of TF domains provide guidelines to examine and articulate "people's interpretive relations with technology" (Orlikowski and Gash 1994, p. 200f).

This article proposes the use of TF as an analytic tool in VSD to support the identification of values using the case of patient accessible electronic health records in Sweden.

\section{The case of patient accessible electronic health records}

In 2012, Region Uppsala in Sweden launched several eHealth services, such as patient accessible electronic health record (PAEHR), appointment booking, renewal of prescriptions, and so forth. Through PAEHR, patients can view a timeline with their personal health information, for example 
diagnoses, lab results, drug prescriptions, and medical notes (Fig. 1). They can also see a log list, which lists all healthcare professionals (HCPs) who accessed their EHR.

All too often patients have not been considered in the design process of EHR systems (cf. Friedman et al. 2006, p. 361). However, in the case of PAEHR in Region Uppsala patients were especially addressed as the direct stakeholders. Unfortunately, here the HCPs were not seen as a "legitimate actor in the development process" (Erlingsdottir and Lindholm 2015, p. 20); probably because they were not regarded as primary users. HCPs use a different system for accessing their patients' EHR, and thus are not using the eHealth service in their professional role. However, the HCPs considered themselves as being affected by PAEHR and reacted strongly against its launch (see e.g., Erlingsdottir and Lindholm 2015). Thus, they should at least be regarded as indirect stakeholders, especially due to their input of record data and potential effects of PAEHR on their work practices. Some physicians even considered changing their use of their professional EHR system, for example, by waiting with documentation after they had talked to the patient (Grünloh et al. 2016). In this case, the patient, as the indirect stakeholder of the professional EHR system, would be affected by that change of practice, because the omitted

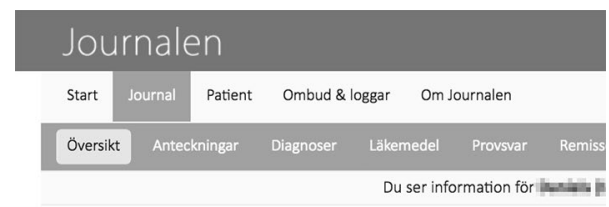

Journalöversikt

|tidsinien ser du ala tillgàngliga uppgitter ur din journal, itdsordning med det nyaste overst. Kilick rad for att se mer detalijer on den. Saknar du nâbon uppgitit?

Vad innebär Nyt//Ovidimerat och Nyt//0signerat?

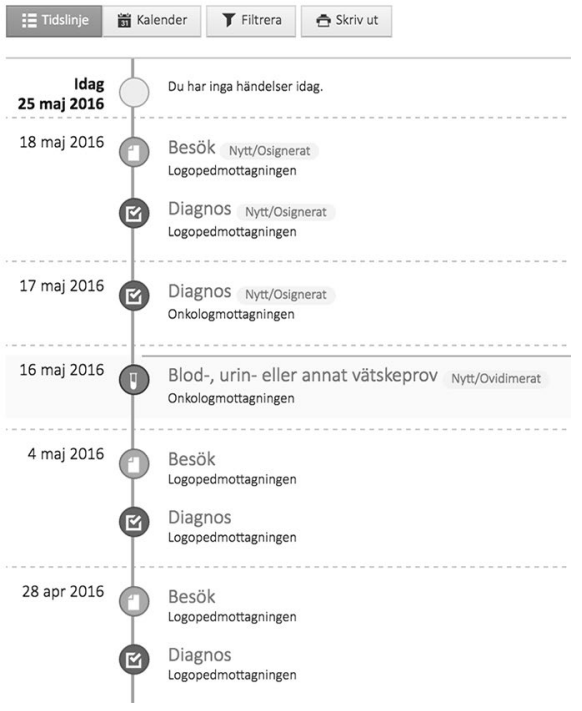

Fig. 1 Screenshot of the system showing the timeline of the health record information would of course not be accessible through PAEHR. To illustrate the complexity of the stakeholder relationship even more, a survey showed that physicians who had used PAEHR as a patient themselves were much more positive towards it (Ålander and Scandurra 2015). Hence, HCPs are initially indirect stakeholders, but become direct stakeholders of PAEHR in case they are also patients. This illustrates, how two related stakeholders (HCPs and patients) can be both direct and indirect stakeholders at different times as they directly use or are indirectly impacted by two different systems.

\section{Related technological frames}

In the case of PAEHR, the stakeholders' needs were in conflict, in that patients were interested in direct access to their up-to-date EHR, while HCPs preferred them to have access only after a delay of 14 days for proofreading (Erlingsdottir and Lindholm 2015). In a qualitative analysis of physicians' TF four main themes were identified: work tool, control, process, and workload (Grünloh et al. 2016). The physicians' assumptions and expectations related to these themes are for example:

- The EHR is their work tool and not supposed to be for the patient.

- Patients would use certain features to monitor their doctors.

- Patients would not understand the content of the records, which would

- lead to undue anxiety,

- an increased workload for the HCPs, and

- disrupt their current work flow, where all information is gathered and discussed among HCPs first before the patient is informed.

Grünloh (2016) related the themes to the aforementioned TF domains (Orlikowski and Gash 1994), as illustrated in Fig. 2.

\section{Related human values}

The qualitative analysis mentioned previously focused on TF and did not explicitly investigate implicated human values. However, TF can be used as an analytic method in VSD, in that the frames can serve as a basis to further identify the corresponding values. The identification of TF can be seen as an activity within both, empirical and technical investigations as the focus is on (a) particular stakeholders, and (b) their interpretation of a particular technology. For this 
Technological Frames

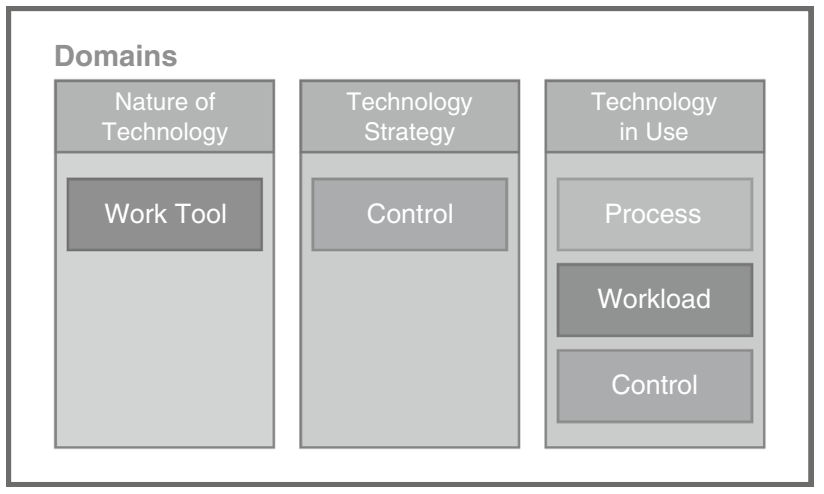

Fig. 2 Main themes related to TF domains (Grünloh 2016, p. 30)

article, the previously identified TF have been re-examined in order to identify corresponding values. In the following, some examples of human values (cf. Friedman and Kahn Jr, 2007) are presented, that may have influenced or informed the HCP's interpretation of PAEHR.

\section{Ownership and property}

Part of the physician's TF was the view of the record as their work tool and hence of themselves as its owner. Thus, giving patients online access may be seen as repurposing their work tool. Many patients, however, regard themselves as the owner and demand access to their health information (see e.g., the "liberate health data" campaign by Campos and Sebastian 2015).

\section{Autonomy}

Part of the TF, particularly in the domain "technology-inuse", was the assumption that giving patients access to their EHR would interfere with the work processes. Patients would be able to read the content even before the HCP had seen them, thus they anticipated the need to "catch up" in less time. Furthermore, physicians were concerned that patient would use PAEHR to monitor the physicians. In this framing, PAEHR conflicts with the professional autonomy of HCPs, who for instance do not want to be pressured to change their work practices or being monitored.

One aim of PAEHR was to enhance patient participation and empowerment by giving patients digital access to their medical information (Erlingsdottir and Lindholm 2015 , p. 23). The human value of autonomy can thus also be related to the personal autonomy of the patient. This could potentially be identified by technological investigations in VSD. However, an essential part of the physicians' TF was that patients would not be able to understand the content and would become unnecessarily anxious. Furthermore, as identified in the TF related to the process, physicians preferred to complete the steps in their process (i.e., interpret test results, make a diagnosis, consult colleagues, determine treatment) before giving that information to the patient (Grünloh et al. 2016). This part of the TF can be related to professional authority, which is in conflict with PAEHR by which patients access their information directly. Katz (2002, p. 85ff) discusses professional authority in that it has been a millennia-long tradition that physicians are the solitary decision maker and thus are reluctant to depart from familiar practices. However, patient-centred care has been defined as being respectful of and responsive to individual patient preferences, which ensures that patient values guide all clinical decisions (Barry and Edgman-Levitan 2012, p. 780). Thus, professional authority implied in the TF conflicts with the personal autonomy of patients.

\section{Privacy and trust}

Providing patients with a log list tackles the issue that HCPs can potentially access them without permission. The idea is that patients easily recognise the names of acquaintances who have no business reading their records. This feature is supposed to support patients' privacy. However, physicians perceived it as a surveillance tool and assumed that patients mistrust them if they read the log list (Grünloh et al. 2016). During the interviews, the physicians referred to their professional expertise and that thus patients should trust them (Grünloh et al. 2016). In this case, interpersonal trust between HCP and patient is perceived to be endangered through a feature for the patient, that is supposed to establish patient's trust in the technology. The endangered "trust in the relationship" was identified through TF, while "trust in the technology" through a particular feature could be identified through technological investigations in VSD.

\section{Human welfare and well-being}

The physicians were concerned that patients could be harmed by reading their EHR (Grünloh et al. 2016). HCPs were also concerned that this system would have a negative effect on the quality of healthcare (Huvila et al. 2013). A study with cancer patients found that direct access to test results can also be crucial for patients' well-being (Rexhepi et al. 2016). The patients described that anxiety was rather caused by having to wait for a long time to receive the results from their physician. They perceived the delay as having a negative impact and thus saw an improvement on their wellbeing by PAEHR (Rexhepi et al. 2016). We can see here that both stakeholders share the same value: the wellbeing of the patient. Thus, the tensions are not among values but among TF, which can then be addressed accordingly. For example, 
one could investigate whether the physicians' frames are based on mere assumptions and whether positive patient experiences can be found also on a large scale.

These examples illustrate tensions between values and thus one has to balance and make trade-offs between them. Tensions may appear in the same value dimension (e.g., autonomy of group A is in conflict with autonomy of group B); or in another value dimension (e.g., personal autonomy of group A conflicts with privacy of group B). In addition, tensions can occur between TF of stakeholders, even if their values concord.

\section{Technological frames as tool in value sensitive design}

It is an ongoing discussion whether human values are universally held or specific to the individual or culture (e.g., Borning and Muller 2012). Friedman and Kahn Jr (2007, p. 1247) proposed a middle ground which allows for an analysis of universal moral values as well as allowing for these values to vary (e.g., depending on culture or context).

Within VSD one can either begin with a particular value, technology, or context of use (Friedman et al. 2006, p. 362). TF can be used as an analytic tool where the context of use is the starting point. The frames conceptualise people's assumptions, expectations, and knowledge, which can be used to examine people's interpretive relations with the technology, and from which corresponding values are then recognised.

TF as an analytical tool can be situated in relation to both empirical and technical investigations. However, it should be emphasised that with TF the technology is investigated from the particular stakeholder's point of view. Instead of the researcher investigating "how existing technological properties and underlying mechanisms support or hinder human values" (Friedman et al. 2006, p. 351), TF include stakeholder's framing. This means that although the technology per se might have been designed to support or at least not hinder certain values, people might experience or frame this quite differently. This was exemplified in the aforementioned log list, which was supposed to protect patient's integrity, but was perceived by HCPs as a surveillance tool. A sole investigation and analysis of the technology may not identify the supported or hindered values as perceived by stakeholders. This way, using TF bridges between empirical and technical investigations in that it not solely focusses on the technology itself but integrates an empirical element.

As proposed by Friedman et al. (2006, p. 365f), value considerations should be integrated into the organisational structure. The aforementioned TF domain Technology Strategy refers to people's view of why their organisation acquired and implemented the technology. By analysing the assumptions and expectations, one may identify not necessarily conflicts between values of organisation and employees, but maybe between organisations' values and how those are perceived by the employees.

Through iterations, the empirical and technological investigations supported by TF can then inform again the conceptual investigations (e.g., by clarifying what a specific value such as well-being means for this particular stakeholder in this particular context).

\section{Conclusion}

As illustrated, several values on an individual and on a group level can be affected by the design and implementation of an eHealth services; both negatively and positively. While many physicians did not see a general value for patients to access their EHR, patients perceived this quite differently. As the examples showed, assumptions, expectations, and experiences of the different stakeholders could be mapped to different or even the same value but with a different perspective, which lead to a different assessment of the usefulness of the system. Using TF in VSD has the potential to support researchers and designers to identify and balance or prioritise the values of different stakeholders. Emotional aspects, such as the strong reaction against PAEHR, are potentially related to or based on values or may even be caused by value tensions. Thus, using TF within VSD also provides an opportunity to enhance our understanding of the relation between emotional aspects and human values, as called for by Sellen et al. (2009).

Acknowledgements Thanks to my supervisor Åke Walldius for the vivid discussions leading to this article. The outlined case study is part of current research within the DOME consortium and has benefited from discussions with other researchers in DOME. Special thanks to Tag Alshehri, Ian S. King, David Hendry, and the anonymous reviewers for their helpful comments and suggestions.

\section{Compliance with ethical standards}

Conflict of interest The author declares that she has no conflict of interest.

Open Access This article is distributed under the terms of the Creative Commons Attribution 4.0 International License (http://creativeco mmons.org/licenses/by/4.0/), which permits unrestricted use, distribution, and reproduction in any medium, provided you give appropriate credit to the original author(s) and the source, provide a link to the Creative Commons license, and indicate if changes were made. 


\section{References}

Ålander, T., \& Scandurra, I. (2015). Experiences of healthcare professionals to the introduction in Sweden of a Public eHealth Service: Patients' online access to their electronic health records. Studies in Health Technology and Informatics, 216, 153-157. https://doi. org/10.3233/978-1-61499-564-7-153.

Barry, M. J., \& Edgman-Levitan, S. (2012). Shared decision makingThe pinnacle of patient-centered care. New England Journal of Medicine, 366(9), 780-781.

Borning, A., \& Muller, M. (2012). Next steps for value sensitive design. In Proceedings of the SIGCHI conference on human factors in computing systems (pp. 1125-1134). New York: ACM.

Campos, H., \& Sebastian, A. (2015). Data liberación: The path to precision medicine. https://www.whitehouse.gov/blog/2015/07/09/ data-liberaci-n-path-precision-medicine, archived at http://www. webcitation.org/6gq7TJ1KM.

Erlingsdottir, G., \& Lindholm, C. (2015). When patient empowerment encounters professional autonomy: The conflict and negotiation process of inscribing an eHealth service. Scandinavian Journal of Public Administration, 19(2), 7-28.

Friedman, B., \& Kahn, P. H., Jr. (2007). Human values, ethics, and design. In A. Sears, \& J. A. Jacko (Eds.), The human-computer interaction handbook (pp 1241-1266). Mahwah: L. Erlbaum Associates Inc.

Friedman, B., Kahn, P. H., Jr., Borning, A. (2006). Value sensitive design and information systems. In P. Zhang, \& D. Galletta (Eds.),
Human-computer interaction and management information systems foundations (pp. 348-372). Armonk: M.E. Sharpe.

Grünloh, C. (2016). To share or not to share? Expectations of and experiences with eHealth services that allow users access to their health information. Licentiate Thesis, KTH Royal Institute of Technology.

Grünloh, C., Cajander, A, \& Myreteg, G. (2016). "The Record is Our Work Tool!" - Physicians' framing of a patient portal in Sweden. Journal of Medical Internet Research, 18(6), e167. https://doi. org/10.2196/jmir.5705. http://www.jmir.org/2016/6/e167/.

Huvila, I., Myreteg, G., \& Cajander, Å (2013). Empowerment or anxiety? Research on deployment of online medical E-health services in Sweden. Bulletin of the American Society for Information Science and Technology, 39(5), 30-33.

Katz, J. (2002). The silent world of doctor and patient, revised edn. Baltimore: Johns Hopkins University Press.

Orlikowski, W. J., \& Gash, D. C. (1994). Technological frames: Making sense of information technology in organizations. ACM Transactions on Information Systems, 12(2), 174-207.

Rexhepi, H., Åhlfeldt, R. M., Cajander, Å, \& Huvila, I. (2016). Cancer patients' attitudes and experiences of online access to their electronic medical records: A qualitative study. Health Informatics Journal, 1-10.

Sellen, A., Rogers, Y., Harper, R. H. R., \& Rodden, T. (2009). Reflecting human values in the digital age. Communications of the ACM, $52(3), 58-66$. 